\title{
Cancer survivors' perspectives and experience on western medicine and traditional Chinese medicine treatment and rehabilitation: a qualitative study
}

\author{
Ji-Wei Wang' \\ Zhi-Qi Yang' \\ Cong Liu' \\ Si-Jia Chen' \\ Qian Shen' \\ Tian-Rui Zhang' \\ Nancy S Partike ${ }^{2}$ \\ Zheng-Ping Yuan ${ }^{3}$ \\ Jin-Ming $\mathrm{Yu}^{\prime}$ \\ 'School of Public Health, Key \\ Laboratory of Public Health Safety, \\ Fudan University, Shanghai, People's \\ Republic of China; ${ }^{2}$ School of Public \\ Health, University of Hawaii at \\ Manoa, Honolulu, HI, USA; ${ }^{3}$ Shanghai \\ Cancer Rehabilitation Club, Shanghai, \\ People's Republic of China
}

\author{
This article was published in the following Dove Press journal: \\ Patient Preference and Adherence \\ 18 December 2014 \\ Number of times this article has been viewed
}

Background: In the People's Republic of China, both western medicine (WM) and traditional Chinese medicine (TCM) are the main treatment and rehabilitation options for cancer patients. This study aimed to explore cancer survivors' perspectives and experience of treatment and rehabilitation, in order to promote patient-centered activities of treatment and rehabilitation.

Methods: Using a qualitative research approach, 68 cancer survivors were recruited from eight community cancer rehabilitation organizations in Shanghai, People's Republic of China. Eight focus group interviews were conducted. All these interviews were transcribed verbatim, and the data were analyzed by theme analysis.

Results: WM was the main choice in treatment phase though study participants noted more side effects. TCM was primarily used in the recovery phase. The lack of communication between doctors and cancer patients appears to affect treatment adherence and impair the doctor-patient relationship. WM was expensive for diagnostic procedures and treatment, while the cumulative costs of frequent use of TCM in the long rehabilitation period were also high. Both treatment options created significant perceived economic burden on patients. Conflicting information about dietary supplements tended to make cancer survivors confused.

Conclusion: Improving the communication between doctors and cancer patients helps to ameliorate cancer patient adherence and the effect of treatments. It is essential to educate cancer patients about the effect and cost of both WM and traditional TCM. Meanwhile, marketing management and guidance to consumers regarding use of dietary supplements in the cancer rehabilitation field are also necessary.

Keywords: preference, adherence, cancer survivor education, focus group interview

\section{Introduction}

The World Health Organization estimated 8.2 million deaths worldwide due to cancer in 2012, and this number was expected to rise to 22 million by the year $2030 .^{1}$ The number of new cancer cases in the People's Republic of China increases by more than 2 million each year and cancer has become the leading cause of death among urban and rural residents. ${ }^{2}$ Significant progress has been made in cancer treatment with the development and promotion of early detection, early diagnosis, and early treatment for cancer, the advent of new chemotherapy drugs, the improvements in surgery and radiotherapy techniques, the use of traditional Chinese medicine (TCM) in cancer treatment and rehabilitation, as well as the diversity of cancer treatment options. ${ }^{3-6}$ All these factors have made more and more cancer patients be able to survive and live better quality lives longer. ${ }^{7,8}$
Correspondence: Jin-Ming Yu School of Public Health, Key Laboratory of Public Health Safety, Fudan University, I 30 Dong-An Road, Shanghai 200032,

People's Republic of China

Tel +862154237868

Email jmy@fudan.edu.cn 
Cancer patients' knowledge and rational choices of cancer treatment and rehabilitation methods play an important role in improving patient adherence to cancer treatment and enhancing their long-term rehabilitation management. ${ }^{9}$ Previous studies have demonstrated that cancer diagnosis caused significant stress on patients, which, along with the lack of professional knowledge, severely affected patients' adherence. ${ }^{10,11}$ Thus, doctor-patient communication in the early stage of treatment is of great importance for patients to preliminarily establish a correct cognitive system of treatment. ${ }^{12}$ In the People's Republic of China, patients generally regard modern medical treatments such as surgery, chemotherapy, and radiotherapy as western medicine (WM), while herbal medicines and acupuncture therapy are seen as TCM treatment. A "push-pull" model suggests that patients seek TCM as a complementary therapy for WM, either because they are dissatisfied with conventional WM (pushed away) and/or because they are attracted (pulled) to complementary therapies of TCM. ${ }^{13}$

A small number of research studies have been conducted to explore cancer survivors' perceptions on the treatment experience in some countries, ${ }^{14,15}$ but there are limited studies from the People's Republic of China. In order to improve cancer patient treatment adherence and enhance the management of cancer rehabilitation, it is necessary to understand the cancer survivors' perspectives of the treatment and rehabilitation experience. Compared to quantitative research, qualitative research can provide more extensive and in-depth information on cancer treatment and rehabilitation from the cancer survivors' point of view. Qualitative research can also help address the limitations of the preset questions in quantitative research, which is helpful to discover new issues and theories. ${ }^{16}$ This study aimed to explore cancer survivors' perspectives on the experience of treatment and rehabilitation through qualitative research, and furthermore provide support for improving patient-centered actions relating to cancer treatment and rehabilitation.

\section{Methods}

\section{Design}

This qualitative study used focus groups to explore cancer survivors' perspectives on the experience of WM and TCM treatment and rehabilitation. The interview guide, consisting of open-ended questions, is presented in Table 1.

\section{Participant recruitment}

Participants were recruited from the Shanghai Cancer Rehabilitation Club (SCRC), Shanghai, People's Republic of China, which is a nongovernment organization exclusively for cancer survivors. Aiming at improving survivors' quality of life, the SCRC regularly offers rehabilitation activities including physical exercise and psychotherapy.

We contacted all 17 branches of the SCRC across Shanghai by email or telephone and eight of them agreed to participate in the study. We sent recruitment advertisements and posters to these eight branches to form one focus group for each branch. A total of 68 consenting cancer survivors participated in the eight focus groups, with six to ten participants in each group. All of these participants were both cancer survivors and members of the SCRC who had taken part in rehabilitation activities in the club. Ethical approval to conduct this study was granted by the Medical Research Ethics Committee of the School of Public Health, Fudan University, Shanghai, People's Republic of China (Protocol number RB \#2013-04-0450).

\section{Focus group}

Eight focus groups were carried out separately in each SCRC branch between November 2013 and December 2013. The purposes and methods of this research were introduced to the participants in detail. All participants provided signed informed consent forms before the focus groups began. Focus groups were led by the first author in either Mandarin Chinese or Shanghai dialect. Free-flowing conversation was encouraged with two other members from the research team ensuring that all questions had been discussed (Qian Shen and Zhi-Qi Yang). All discussions were recorded using hand-written notes, audio, and video (Tian-Rui Zhang). Each interview lasted 60-90 minutes. Participants' demographics were obtained from a preinterview questionnaire. Participants were provided an incentive of 20 yuans at the end of focus group.

Table I Interview questions

I. What influence did the treatment and rehabilitation have upon your health and/or disease?

2. What kind of treatment and rehabilitation did you feel that you needed?

3. What kind of treatment and rehabilitation have you received?

4. What did you think of the traditional Chinese medicine's effects on your personal experiences with cancer treatment and rehabilitation?

5. What did you think of the western medicine's effects on your personal experiences with cancer treatment and rehabilitation?

6. What is the most impressive thing you have experienced in the cancer treatment and rehabilitation?

7. Do you have any further comments? 


\section{Data analysis}

The video recordings were transcribed verbatim and checked back against the hand-written notes and the audio recordings (Zhi-Qi Yang and Cong Liu). After reading the transcriptions multiple times to promote detailed familiarity with their contents, primary coding was conducted using thematic analysis (Zhi-Qi Yang). This coding was collated to generate potential themes which were checked back against the original data (Si-Jia Chen). As a preliminary validation exercise, these themes were reviewed and cross-checked against the transcriptions (Qian Shen). Finally, the whole research team came together to discuss and decide on the final themes, including resolving any discrepancies identified in the initial rounds of thematic analysis. When all the themes, patterns, and connections were determined, they were discussed and reviewed by all authors to ensure accuracy and form the final themes. ${ }^{17,18}$ As it is very essential to guarantee the accuracy of the participants' quotes when translated from Mandarin to English, a researcher (Jiang Li) who spoke both Mandarin and English checked the translation several times to ensure the greatest possible equivalence between the translation and source text.

\section{Results}

Sixty-eight participants including 18 males $(26 \%)$ and 50 females $(76 \%)$ were adults between the ages of 42 and 78 years. Most (76.47\%) of the participants have lived for 5 years or more after cancer diagnosis. Participants' demographics are summarized in Table 2.

During the analysis, two themes were identified: perspectives on the WM treatment and perspectives on the TCM treatment and rehabilitation.

\section{Perspectives on WM treatment}

\section{Treatment}

WM was the participants' main choice of cancer treatment during the treatment phase

When asked about the choice of treatment means, study participants indicated that after diagnosis, they had been given comprehensive therapies combining various WM which included local treatment (eg, surgery and radiotherapy) and systemic treatment (eg, chemotherapy and biological therapy).

We had to listen to the doctor in terms of surgery and chemotherapy. If my doctor asked for five times of chemotherapy, I was not allowed to miss (the chemotherapy) even once. [n5fg8, male, colorectal cancer]

Cancer survivors believed that the WM curative effect varied from person to person, and was related to individual
Table 2 Demographic characteristics of the cancer patients interviewed $(\mathrm{n}=68)$

\begin{tabular}{|c|c|}
\hline Characteristics & n (\%) \\
\hline \multicolumn{2}{|l|}{ Age range (years) } \\
\hline $42-49$ & $4(5.88)$ \\
\hline $50-59$ & $26(38.24)$ \\
\hline $60-69$ & 31 (45.59) \\
\hline $70-78$ & $7(10.29)$ \\
\hline \multicolumn{2}{|l|}{ Sex } \\
\hline Male & $18(26.47)$ \\
\hline Female & $50(73.53)$ \\
\hline \multicolumn{2}{|l|}{ Education } \\
\hline Junior high school or low & $25(36.76)$ \\
\hline Senior high school & $24(35.29)$ \\
\hline Junior college and tech college & II (16.18) \\
\hline University & $8(11.76)$ \\
\hline \multicolumn{2}{|l|}{ Tumor } \\
\hline Breast cancer & 31 (45.59) \\
\hline Colorectal and rectal cancer & $10(|4.7|)$ \\
\hline Lung cancer & $7(10.29)$ \\
\hline Leukemia & $6(8.82)$ \\
\hline Lymphoma & $4(5.88)$ \\
\hline Thyroid cancer & $3(4.4 I)$ \\
\hline Liver cancer & $2(2.94)$ \\
\hline Ovarian cancer & $2(2.94)$ \\
\hline Tongue cancer & I (I.47) \\
\hline Nasopharyngeal cancer & I (I.47) \\
\hline Endometrial cancer & I (I.47) \\
\hline \multicolumn{2}{|l|}{ Time since diagnosis (years) } \\
\hline$<1$ & I (I.47) \\
\hline $1-2$ & $4(5.88)$ \\
\hline $3-4$ & $11(16.18)$ \\
\hline$\geq 5$ & $52(76.47)$ \\
\hline \multicolumn{2}{|c|}{ Monthly household income (Chinese yuan) } \\
\hline Up to 2,000 & $15(22.06)$ \\
\hline $2,001-3,000$ & $20(29.4 I)$ \\
\hline $3,001-4,000$ & $139(19.12)$ \\
\hline $4,00 \mathrm{I}-5,000$ & $13(19.12)$ \\
\hline$>5,000$ & $7(10.29)$ \\
\hline
\end{tabular}

physical status, psychological status, therapeutic regimen, and many other factors.

It (the WM curative effect) is not clear. In one case, the patient did not take any medicine. As a result, the cancer has spread. For those of us who have been taking medicine, the cancer hasn't spread. [n7fg3, female, breast cancer]

\section{Treatment side effects}

Study participants indicated that local pain at the surgical site and postoperative complications were the major side effects of their surgeries. Chemotherapy caused more adverse effects on patients' appearance, physiological and psychological well-being. Some participants pointed out that the side effects of chemotherapy were extreme pain that was beyond 
imagination, which has become the main reason why they were forced to suspend the chemotherapy.

The physiological reaction of chemotherapy was very severe, such as hair loss, blood vessels turning black, vomiting, and being unable to eat anything. [n1fg6, male, colorectal cancer]

Patients with breast cancer or ovarian cancer indicated that physiological and appearance changes after WM treatment made them lose their femininity, which brought trouble to their daily life and conjugal relationship, and made them have a sense of shame and self-abasement.

I would like to say that I felt sorry to my husband after I had this disease. I mean sex, I was so sorry. To be honest, we rarely made love to each other after I had cancer. [n5fg1, female, breast cancer]

\section{Communication with the doctors}

\section{Good communication}

Some study participants expressed that they felt their doctors were kind and friendly and that patients should respect and trust their doctors. Communicating with doctors actively with open exchanges back and forth, expressing patients' concerns and preferences for treatment was extremely important for doctors to make the best decisions on the therapeutic regimen.

I would tell the doctor how and where I felt the pain, because I was the one who has the disease, not the doctor... so the doctor could make a good plan for me based on his expertise. It would be easier for him to address a medical problem after getting enough information. Normally, he would give you a better solution. [ $\mathrm{n} 4 \mathrm{fg} 3$, female, breast cancer]

\section{Lack of communication}

A few study participants considered doctors as self-centered, which meant they could not understand patients' pain and consider it from the patient's point of view. Due to the doctors' dominant position and huge patient load in the hospital, patients often had various difficulties in acquiring enough professional advice on cancer treatment and control from their doctors. Lack of time, high cost, indifference, and rejecting other alternative treatments were cited as barriers of doctor-patient communication.

Generally, I'd like to see my own doctor. If I went to other hospitals and talked to another doctor, he would be impatient. So, I'm not willing to change my doctor. As patients, we are very anxious, and we hope for more communication. But the doctor might think that you've had a surgery and you come here only for medicine. So they would not be sincere, and I don't want to go there. [n1fg2, male, colorectal cancer]

\section{Adherence to the doctor's advice}

Due to the barriers in the patient-doctor communication, cancer survivors might have doubts on the curative effects, overtreatment, and the lack of individualized regimen. As a result, cancer patients' adherence to doctors' advice may be compromised.

The doctor was just not listening to you, he had his plan, the only one plan. He would never ask about the severity or what kind of treatment you wanted. Never. I think he should consider of my personal opinion. There is one regime if I didn't have a serious pain, while there is another one if I did. [n4fg6, female, breast cancer]

\section{Expenses}

Expenses of surgeries and drugs

Study participants expressed that the WM had more medical expenses in the early stage of cancer treatment, in comparison to the TCM treatment. The cost and availability of the imported western equipment and medicine varied and greatly increased the treatment costs.

Domestic and imported drugs are actually the same. I read a piece of newspaper yesterday, which said that an anti-breast cancer drug cost 24,000 in Shanghai and only 8,000 yuan in Hong Kong. They would like to buy it from Hong Kong because of the big difference in price. It is the import tax in Shanghai that makes the imported drugs so expensive. [n2fg8, male, gastric cancer]

\section{Expenses of medical examination}

Study participants were always afraid of cancer recurrence and metastasis. They would see a doctor for a related medical examination immediately, if they felt even slightly uncomfortable. Patient anxiety and the need to reassure themselves resulted in a large amount of medical examination expenses.

I have had cancer for a decade. I have to spend over one thousand yuan for a systemic check-up each time. But my salary is just about 2,000 yuan a month. How can I live? 
It would be better if the government medical insurance covered some medical examination expenses. Sometimes even a little feeling of tiredness that would make me hurry to hospital, and it would cost me another 1,000 yuan. [n1 fg2, male, colorectal cancer]

\section{Long-term expenses}

Study participants described that the reimbursement rate of government medical insurance did not meet their needs. They said that the government medical insurance covered an inadequate length of time with 2 years for the WM and 5 years for the TCM, and had a limited scale, which excluded some specific medicine, imported medicine, examinations of diagnose, and follow-up. Furthermore, the procedure of reimbursement was complicated for patients to understand.

This is a lifetime disease. Government medical insurance used to cover 2 years, now covering 5 years. But 5 years is still not enough. And it only included Chinese medicine, without western medicine. The medical examinations alone cost thousands of yuan of insurance, so it's not enough. [n6fg4, female, breast cancer]

\section{Perspectives on the TCM treatment and rehabilitation \\ Treatment}

Chinese medicine was the main choice in rehabilitation phase

The study participants chose the TCM as the major complementary therapy for physical conditioning in rehabilitation phase, and this often lasted for a long time.

It has been 12 years after I had the surgery. I have never caught a cold because I have insisted on taking Chinese medicine all these years. As for Chinese medicine, an example comes from my mom. She was diagnosed with gastric cancer at the age of 70. From then on, she began to take Chinese medicine. She has taken for years and now she is 100 years old. [ $\mathrm{n} 4 \mathrm{fg} 5$, male, colorectal cancer]

\section{Treatment effects}

Most study participants thought that long-term use of the TCM benefited them in rebuilding the immune system which was destroyed by cancer and treatment and that the TCM could prevent the recurrence and metastasis of cancer. Some study participants thought that the TCM could not bring obvious short-term efficacy and doubted that they were just placebos. But the fear of death promoted them to try any complementary therapies that might have some benefits. Besides, almost all the participants agreed TCM was a harmless and noninvasive method of treatment.

Our immune system has been destroyed. I was diagnosed with bony metastases this year. I didn't have any other treatments except for Chinese medicine. I went to see a wellknown Chinese medicine doctor and took Chinese medicine after I was diagnosed with cancer in June. I reexamined in October, and the tumor was the same as what it was in June. It didn't become worse. [n3fg4, female, breast cancer]

\section{Communication with the doctors}

Most study participants indicated that the TCM doctors were more friendly and more willing to listen to patients' opinions, when compared to the WM doctors. Their inquiries included more details and they paid more attention to the patients' daily life, such as diet, exercise, and the relationship with families. A longer process of diagnosis and treatment provided more opportunities for doctor-patient communication.

Compared with western medicine doctors, the Chinese medicine ones prefer communicating with you. The four diagnostic methods of traditional Chinese medicine demand doctors to ask for more details. [n8fg2, female, breast cancer]

\section{Expenses}

Long-term or even lifetime use of the TCM was recommended for the cancer survivors. However, the government medical insurance on the TCM has a time constraint of 5 years, which resulted in cumulative medical costs that cause huge economic burdens on the patients.

The government medical insurance covers 5 years for Chinese medicine and 2 years for western medicine. Sometimes we want to take Chinese medicine for improving our conditions, but we can't. We have to pay for the medicine, which is very expensive. But we don't have enough income. If you can let us keep taking Chinese medicine for free, we will have less pressure. Otherwise, we can't afford the medicine even if we want to take it. [n1fg2, male, colorectal cancer]

\section{Dietary supplements}

Some participants thought dietary supplements had certain benefits, so they would buy some, according to their health and economical condition in the rehabilitation phase of their 
cancer illness. Study participants said that a large number of dietary supplements contained false advertisements and were profit-driven rather than evidence-based, which always confused and were often confusing in the concepts and efficacy of TCM and dietary supplements. Some cancer survivors could not distinguish between dietary supplements and TCM.

I would read the instruction of the product. If it could increase my immunity or had something to do with my disease, then I would take some. But just for a year or two. [n3fg8, female, breast cancer]

The dietary supplements are all described as they can heal any disease, including cancer. And we don't believe that. Even if we believe it, we can't afford to buy every product which costs thousands of yuan. [n10fg8, female, lymphoma]

\section{Discussion}

Our findings indicated that the WM was the main choice in treatment phase, though study participants noted more side effects. TCM was primarily used in the recovery phase. The lack of communication between doctors and cancer patients appears to affect treatment adherence and impair the doctorpatient relationship. WM was expensive for diagnostic procedures and treatment, while the cumulative costs of frequent use of TCM in the long-term rehabilitation period were also high. Both treatment options created a huge financial burden on patients. Conflicting information on dietary supplements tended to make cancer survivors confused.

Cancer survivors' adherence and options to treatment depends on their knowledge of cancer treatment. ${ }^{14}$ Physiological adverse effects caused by the WM treatment methods such as surgery, radiotherapy, and chemotherapy make it difficult for patients to recover to a physical and psychological stable state quickly. ${ }^{19}$ For many cancer survivors, the fear of side effects of treatment was identified as the leading reasons why patients quit part of or the whole treatment plan. ${ }^{20}$ Diagnosis and treatment of the WM are based on the established scientific theories. The assured diagnosis and sure curative effects are more important for patients, rather than rebuilding the steady state of the body. As a consequence, the WM treatment was considered as the first choice of patients in the early stage of cancer treatment.

Participants of this study generally believed in TCM in the rehabilitation phase; especially those who experienced ineffective chemotherapy or were forced to quit because of severe side effects, preferred TCM which is less risky.
But previous studies indicated that TCM has not been proven to have significant effects on reducing the side effects of WM. ${ }^{21}$ Most participants thought it would take a long time before TCM worked, which is different from previous studies. $^{22}$ The lack of knowledge about curative effects and patients being more involved in decision-making process increase the cancer patients' burden in making decisions on treatment options. More clinical research needs to be conducted to confirm the effects of long-term use of TCM, as well as the toxicity and side effects it causes. It could provide evidence to conduct a cancer patient education program on TCM. With complicated information about dietary supplements in the field of cancer rehabilitation flooding the market, it is difficult for patients to discern between these products which may lead to unknowing/uneducated consumption. It is essential to enhance marketing management and consumption guidance geared toward better patient education. TCM was considered as one complementary and alternative medicine (CAM) which is increasingly being used in many countries, especially in East Asian countries and areas. ${ }^{23-25}$ Our study indicated that using TCM was not only a medical issue, but also involved a biopsychosocial interaction between patients, physicians, and the medical system, as previously reported for other CAMs. ${ }^{26,27}$ In our study, the TCM was primarily used in the cancer recovery phase and both TCM and WM are not cheap. The patient choice of CAM in the different phases of cancer treatment and rehabilitation was often overlooked in the previous studies.

It is crucial for doctors to cope with the cancer patients' adverse effects during the cancer treatment. However, patients' anxiety experienced before the treatment is graver than that in any other stage of the treatment. ${ }^{28}$ Doctors in their dominant position of authority may intimidate patients' communication, thus impacting the ability to assess and address patient concerns in a timely manner. Generally, if doctors concentrate on the disease itself but neglect the humanistic side of care, this may result in patients' decreasing adherence. ${ }^{29}$ During the establishment and implementation of the treatments, the communication between doctors and cancer patients should be strengthened. Doctors are required to inform cancer patients of their treatment options, the pros and cons of the treatment options, and allow patients to choose the best option for their unique situation. In addition, a patient-centered plan for treatment and rehabilitation should be emphasized. It is necessary for medical staff to be trained in better communication skills to adjust the inequality between patients and doctors. They are also expected to offer more psychological support, satisfy cancer patients' 
need for health information on their diseases, and provide comprehensive health care services.

As a chronic disease, cancer survivor self-management is the major mode of the cancer management. ${ }^{30,31}$ Only by constantly improving patients' knowledge for treatment and rehabilitation can they cope with various issues emerging in the long-term treatment and rehabilitation process..$^{32}$ Patients should keep a positive proactive attitude toward their own health problems. They should also participate in the planmaking process and display their subjective initiative. ${ }^{33}$ At the same time, patients need to show their respect and trust doctors. Good communication between doctors and patients provides practical and effective information with high pertinence, which contributes to improving doctorpatient relationship and patients' adherence to treatments. Meanwhile, it is beneficial to manage cancer treatment and rehabilitation scientifically.

The financial burden of cancer treatment expenses has become one of the most significant obstacles for most cancer patients to seek effective therapies. However, better insurance coverage for treatment options may improve cancer patients' adherence to treatment. ${ }^{14}$ Cancer survivors in this study reflected a belief that the more expensive medicine would be more effective and curative, which is why they chose more expensive imported medicine instead of the TCM option initially. However, cancer survivors tended to choose the TCM model in the rehabilitation phase. Therefore, health care policies and insurance plans should take cancer patients' multiple choice of medicines and the long-term rehabilitation into full consideration.

\section{Limitations}

The conclusions that can be drawn from this study are limited to the extent that participants were all recruited from a cancer rehabilitation club. Women accounted for the majority of participants, which might have something to do with women's active role in social activities and cancer rehabilitation organizations in nature. Finally, we did not allocate participants to different focus groups based on the type of cancer (almost one-half had breast cancer) which undermines the ability to detect subtle differences in treatment and rehabilitation experiences of cancer survivors living with different diagnoses. In addition, different cancers may also differ in cost, dietary regulation and supplementation, and other experiences; yet, it is really hard to reach a conclusion based on the small sample size in this study. Therefore, it is necessary to investigate the percentage of cancer survivors and patients who had similar issues as reported by the study participants by socioeconomic status, the type of cancer, and its treatment in the next quantitative study.

\section{Conclusion}

Understanding cancer survivors' perspectives and experience on the WM and TCM treatment and rehabilitation will contribute to improve cancer patients' and survivors' adherence to treatment regimen, which can promote patient-centered activities of treatment and rehabilitation to be carried out effectively. First, improving the communication between doctors and cancer survivors contributes to ameliorate cancer survivor adherence and the effect of treatments. Second, it is essential to develop a comprehensive and holistic cancer survivor education program on both WM and TCM; especially on the side effects of WM and the treatment effects of TCM. Third, marketing management and guidance to consumers regarding use of dietary supplements in cancer rehabilitation field are also necessary.

\section{Acknowledgments}

This study was supported by grants from the National Social Science Foundation of China (Award No 13CSH076) and the Shanghai Municipal Commission of Health and Family Planning (Award No 20134068).

\section{Disclosure}

The authors report no conflicts of interest in this work.

\section{References}

1. Ferlay J, Soerjomataram I, Ervik M, et al. GLOBOCAN 2012 v1.0, Cancer Incidence and Mortality Worldwide: IARC Cancer Base No 11 [Internet]. Lyon, France: International Agency for Research on Cancer; 2013. Available from: http://globocan.iarc.fr.

2. Wang Y, Wei L, Liu J, Li S, Wang Q. Comparison and analysis of the incidence and mortality rate of cancer in developed and developing countries. Chin J Clin Oncol. 2012;39:679-682.

3. Madan RA, Arlen PM. Recent advances revolutionize treatment of metastatic prostate cancer. Future Oncol. 2013;9:1133-1144.

4. Yin L, Hu Q, Hartmann RW. Recent progress in pharmaceutical therapies for castration-resistant prostate cancer. Int J Mol Sci. 2013; 14:13958-13978

5. Psutka SP, Feldman AS, McDougal WS, McGovern FJ, Mueller P, Gervais DA. Long-term oncologic outcomes after radiofrequency ablation for T1 renal cell carcinoma. Eur Urol. 2013;63:486-492.

6. Lee YW, Chen TL, Shih YR, et al. Adjunctive traditional Chinese medicine therapy improves survival in patients with advanced breast cancer: a population-based study. Cancer. 2014;120:1338-1344.

7. Gu J, Cao X. Conceptual consideration of cancer, challenges and opportunities for cancer biotherapy. Chin J Cancer Biother. 2008;15:2-7.

8. Veronesi U, Boyle P, Goldhirsch A, Orecchia R, Viale G. Breast cancer. Lancet. 2005;365:1727-1741

9. Lim JW, Gonzalez P, Ming F, Letzkus W, Kimlin T, Giwa A Understanding the cultural health belief model influencing health behaviors and health-related quality of life between Latina and AsianAmerican breast cancer survivors. Support Care Cancer. 2009;17 $1137-1147$. 
10. The National Comprehensive Cancer Network. Distress Management (Version 1.2009). NCCN Clinical Practice Guidelines in Oncology. Available from: https//www.cancerlearning.gov.au/docs/distress.pdf. Accessed January 20, 2010.

11. Zabora J, BrintzenhofeSzoc K, Curbow B, Hooker C, Piantadori S. The prevalence of psychological distress by cancer site. Psychooncology. 2001; 10:19-28.

12. Coyne E, Borbasi S. Living the experience of breast cancer treatment: the younger women's perspective. Aust J Adv Nurs. 2009;26:6-13.

13. Kelner M, Wellman B. Health care and consumer choice: medical and alternative therapies. Soc Sci Med. 1997;45:203-212.

14. Maryam F, Mohamed AH, Aishah KS, Asrul AS, Tan BS, Muhammad AF. A qualitative exploration of Malaysian cancer patients' perspectives on cancer and its treatment. BMC Public Health. 2011;11:525.

15. Smithson J, Britten N, Paterson C, Lewith G, Evans M. The experience of using complementary therapies after a diagnosis of cancer: a qualitative synthesis. Health (London). 2012;16:19-39.

16. Berg BL. Qualitative Research Methods for the Social Sciences. 4th ed. Boston. Allyn and Bacon; 2001.

17. Braun V, Clarke V. Using thematic analysis in psychology. Qual Res Psychol. 2006;3(2):77-101.

18. Joffe H, Yardely L. Content and thematic analysis. In: Marks DF, Yardley L, editors. Research Methods for Clinical and Health Psychology. London, UK: Sage Publications Ltd; 2003:56-68.

19. Korfage IJ, Hak T, Koning HJ, Essink-Bot ML. Patients' perceptions of the side-effects of prostate cancer treatment-a qualitative interview study. Soc Sci Med. 2006;63:911-919.

20. Verhoef MJ, White MA. Factors in making the decision to forgo conventional cancer treatment. Cancer Pract. 2002;10:201-207.

21. Mok TS, Yeo W, Johnson PJ, et al. A double-blind placebo-controlled randomized study of Chinese herbal medicine as complementary therapy for reduction of chemotherapy-induced toxicity. Ann Oncol. 2007;18:768-774.

22. Xu W, Towers AD, Li P, Collet JP. Traditional Chinese medicine in cancer care: perspectives and experiences of patients and professionals in China. Eur J Cancer Care (Engl). 2006;15:397-403.
23. Yamashita H, Tsukayama H, Sugishita C. Popularity of complementary and alternative medicine in Japan: a telephone survey. Complement Ther Med. 2002;10(2):84-93.

24. Lee SI, Khang YH, Lee MS, Kang W. Knowledge of, attitudes toward, and experience of complementary and alternative medicine in western medicine- and oriental medicine-trained physicians in Korea. Am J Public Health. 2002;92(12):1994-2000.

25. Liu CH1, Tang WR, Wang HM, Lee KC. Cancer patients' experience of combined treatment with conventional and traditional Chinese medicine: a biopsychosocial phenomenon. Cancer Nurs. 2011;34(6):495-502.

26. Ben-Arye E, Bar-Sela G, Frenkel M, Kuten A, Hermoni D. Is a biopsychosocial-spiritual approach relevant to cancer treatment? A study of patients and oncology staff members on issues of complementary medicine and spirituality. Support Care Cancer. 2006;14(2):147-152.

27. Chan C, Ho PS, Chow E. A body-mind-spirit model in health: an Eastern approach. Soc Work Health Care. 2001;34(3-4):261-282.

28. Chernecky C. Temporal differences in coping, mood and stress with chemotherapy. Cancer Nurs. 1999;22:266-276.

29. Mellon S, Berry-Bobovski L, Gold R, Levin N, Tainsky MA. Communication and decision-making about seeking inherited cancer risk information: findings from female survivor-relative focus groups. Psychooncology. 2006;15:193-208.

30. Fisher EB, Brownson CA, O'Toole ML, Shetty G, Anwuri VV, Glasgow RE. Ecological approaches to self-management: the case of diabetes. Am J Public Health. 2005;95:1523-1535.

31. McCorkle R, Ercolano E, Lazenby M, et al. Self-management: enabling and empowering patients living with cancer as a chronic illness. CA Cancer J Clin. 2011;61:50-62.

32. Bodenheimer T, Lorig K, Holman H, Grumbach K. Patient selfmanagement of chronic disease in primary care. JAMA. 2002;288: 2469-2475

33. Caspi O, Koithan M, Criddle MW. Alternative medicine or "alternative" patients: a qualitative study of patient-oriented decision-making processes with respect to complementary and alternative medicine. Med Decis Making. 2004;24:64-79.
Patient Preference and Adherence

\section{Publish your work in this journal}

Patient Preference and Adherence is an international, peer-reviewed, open access journal that focuses on the growing importance of patient preference and adherence throughout the therapeutic continuum. Patient satisfaction, acceptability, quality of life, compliance, persistence and their role in developing new therapeutic modalities and compounds to optimize

\section{Dovepress}

clinical outcomes for existing disease states are major areas of interest for the journal. This journal has been accepted for indexing on PubMed Central. The manuscript management system is completely online and includes a very quick and fair peer-review system, which is all easy to use. Visit http://www. dovepress.com/testimonials.php to read real quotes from published authors. 